Genome Analysis

\title{
Rapid molecular evolution in a living fossil
}

\author{
Jennifer M. Hay ${ }^{1}$, Sankar Subramanian ${ }^{1}$, Craig D. Millar ${ }^{2}$, Elmira \\ Mohandesan $^{1}$, David M. Lambert ${ }^{1^{*}}$
}

${ }^{1}$ Allan Wilson Centre for Molecular Ecology and Evolution, Institute of Molecular BioSciences, Massey University, Private Bag 102904 NSMC, Auckland, New Zealand

${ }^{2}$ Allan Wilson Centre for Molecular Ecology and Evolution, School of Biological Sciences, University of Auckland, Private 92019, Auckland, New Zealand

* Corresponding author: Lambert, D.M. (D.M.Lambert@massey.ac.nz) 


\begin{abstract}
The tuatara of New Zealand is a unique reptile that coexisted with dinosaurs and has changed little morphologically from its Cretaceous relatives. Tuatara have very slow metabolic and growth rates, long generation times and slow rates of reproduction. This suggests that the species is likely to exhibit a very slow rate of molecular evolution. Our analysis of ancient and modern tuatara DNA shows that, surprisingly, tuatara have the highest rate of molecular change recorded in vertebrates. Our work also suggests that rates of neutral molecular and phenotypic evolution are decoupled.
\end{abstract}

\title{
The uniqueness of tuatara
}

The tuatara (Sphenodon punctatus) found only in New Zealand, is the last surviving member of a distinct reptilian order Sphenodontia that lived alongside early dinosaurs and separated from other reptiles in the Upper Triassic more than 220 million years ago $[1,2]$. The skeletal morphology of modern tuatara show a remarkable similarity to some Cretaceous sphenodontian fossils [1] which has led to them being described as a "living fossil” [2]. In addition to their stable morphology, tuatara are characterized by several distinct physiological features that are uncommon among reptiles. They have a low body temperatures (they are active down to $5^{\circ} \mathrm{C}$ in the wild); slow rates of growth $(\sim 50 \mathrm{~cm}$ in 35 years); a slow metabolism; a long generation time (sexual maturity at 10-15 years); and a slow reproductive rate (at 2-5 year intervals) [3-5]. It has been argued that generation time, metabolic rate, body temperature and body size [6, 7] modulate the rate 
of neutral molecular evolution. In tuatara, the rare combination of slow rates of morphological evolution, growth and metabolism suggest that rates of neutral DNA sequence evolution should be slow. To examine this we have sequenced the mitochondrial DNA hypervariable regions (HVR) of the control region from the modern and known-age ancient tuatara individuals and estimated the rate of evolution of this region.

\section{Estimation of molecular evolutionary rate using ancient DNA methodology}

Traditionally rates of molecular evolution have been estimated using phylogenetic

methods, in which levels of genetic divergence among closely related species have been calibrated against time points in the fossil record. However, this is not possible in tuatara as there are no closely related species, one of the characteristics of 'living fossils'.

Instead, we have recovered nucleotide sequences from known-age sub-fossil bones of tuatara and using Bayesian statistics, we have estimated the rate of molecular evolution of the mitochondrial hypervariable regions.

We have amplified and sequenced mtDNA sequences from sub-fossil bones of 33 ancient tuatara and from blood samples of 41 modern individuals covering the range of modern populations and ancient sites (for locations of modern and ancient samples and methods see online supplementary material). We had 29 bones radiocarbon dated and inferred dates of four more from a well stratified site; the ages of the bones range from 650 - 8750 years before present. A 468 bp DNA sequence (including alignment gaps) from the mitochondrial control region (HVR I and HVR II) was recovered in three nonoverlapping fragments from the ancient samples and a 1039 bp sequence covering the 
control region and flanking tRNAs from the modern populations, but we used just the 468 bp sequenced in the ancient samples in these analyses (Genbank Accession numbers xxxxx-yyyyy). We used Bayesian statistics based on a Markov chain Monte Carlo (MCMC) approach to estimate the evolutionary rate of the HVR sequences (online supplementary material). The rate estimate was obtained using a general time-reversible model of evolution. To account for the rate variation among nucleotide positions we used a discrete gamma distribution of six rate categories. The rate was estimated assuming a constant population size and a relaxed uncorrelated log-normal molecular clock.

\section{Higher rate of molecular evolution in tuatara}

Our analysis shows that the mitochondrial HVR regions of tuatara evolve at a rate of 1.56 (HPD 0.83 - 2.34) substitutions per nucleotide per million years. This estimate remained almost the same when modifying a range of prior assumptions such as substitution model, molecular clock and population growth (online supplementary material). In additional analyses, we also used only the nucleotides present in all the ancient and modern sequences (complete site deletion) and we excluded the first four nucleotides that contain two distinct motifs (see online supplementary material). This produced a conservative rate estimate of 1.37 (HPD 0.66 - 2.2). Generally, evolutionary rates obtained using traditional calibration methods, estimate HVR rates at $\sim 0.2$ substitutions per bp per MY $[8,9]$. This estimate is almost an order of magnitude lower than those estimated using ancient DNA methods $[10,11]$. Because the rates estimated by calibration are consistently lower than those estimated from ancient DNA, we compared our result only with previous studies (Figure 1) using the latter approach. The rate estimates in Figure 1 
show that the evolutionary rate of the tuatara HVR regions is the highest among the vertebrate species studied to date. This rate is $>50 \%$ faster than that of other vertebrates (Figure 1). Post-mortem ancient DNA damage can cause nucleotide changes that increase the estimate of the rate of molecular evolution [12-15], as could sequencing ancient DNA Numts instead of mtDNA. However, neither can be invoked to explain the high rate of evolution in tuatara because of the observed similarities in ancient and modern HVR sequence, e.g., in nucleotide composition and nucleotide pair frequencies (online supplementary material). Recent studies show that the vast majority of the ancient DNA damage results in $\mathrm{C} \rightarrow \mathrm{T}$ changes $[16,17]$. To examine this we identified the orientation of all the nucleotide changes in ancient and modern HVR sequences using a maximum likelihood approach implemented in the software PAML [18]. We found that the ratio of $\mathrm{C} \rightarrow \mathrm{T}$ to $\mathrm{T} \rightarrow \mathrm{C}$ changes in the modern and ancient populations were almost identical (2.71 and 2.66 respectively, see online supplementary material table S4). Furthermore, the sites of nucleotide substitution are the same in ancient and modern samples (online supplementary material Fig. S3). There are seven sites where there is variation only in multiple ancient and no modern samples, but in each case the individuals are in the same geographic location suggesting geographic variation rather than miscoding lesions or Numts. A corroborating factor is that in phylogenetic tree analyses (not shown), branch lengths to ancient samples are not consistently longer than to modern samples. Ancient samples are from locations both further north (warmer climate) and south (cooler) of modern populations, but there are no apparent north to south or any other geographic or climatic cline in mutation patterns to indicate environmentally selected variation rather than neutral evolutionary change. These factors 
further confirm that there was minimal post-mortem damage to the DNA, that the ancient sequences obtained are authentic, and the unexpectedly high tuatara evolutionary rates are real. .

\section{Neutral evolution and life history traits of tuatara}

Given this high rate of molecular evolution, the stable morphology of tuatara over tens of millions of years is remarkable. By contrast, the birds and mammals shown here have undergone considerable morphological change over the same period. The higher rate of tuatara HVR evolution does not seem to be the result of a lineage specific effect because the rate of evolution in other reptiles is not higher than those recorded in birds and mammals [19]. The observed higher rate of tuatara HVR evolution does not support the hypothesized inverse relationship between generation time and evolutionary rates [20], as tuatara have a much longer generation time (10-15 years) than do birds and mammals (< 6 years). Our results also contradict the notion that poikilothermic (cold-blooded) animals, such as tuatara, should have slower rates of neutral molecular evolution than homeothermic (warm-blooded) animals, such as mammals and birds [6]. Other life history traits such as body mass and metabolic rate cannot explain the elevated rate of evolution in tuatara. The slow metabolic rate of tuatara compared to other vertebrates suggests that the reported positive relationship between metabolic rate and molecular

evolutionary rate cannot be generalized [6, 7]. Furthermore, the rate of HVR evolution in tuatara is not significantly different from the rates obtained for penguin, aurochs or moa. This result argues against the effect of body mass on the rate of nucleotide evolution [6, 
7], as the body masses of the vertebrates shown in Figure 1 vary up to three orders of magnitude (1-1000kg).

\section{Concluding remarks}

Previous studies on living fossils such as the coelacanth and the horse shoe crab have suggested a substantial nucleotide diversity in these phylogenetically distinct species [21, 22], perhaps indirectly suggesting a high evolutionary rate. However this is the first study to directly estimate the rate of evolution in a living fossil and also the first attempt to quantify the neutral evolutionary changes in a species having such extreme physiological and life history traits. The results of our study support the hypothesis that rates of neutral molecular and phenotypic evolution are decoupled [23]. Hence, life history and physiological parameters do not explain all differences in rates of neutral molecular evolution. Even if rates of evolution are decoupled in nuclear and mitochondrial DNA, metabolic rate theories [6,7] have suggested higher temperatures may cause more oxidative damage in the mitochondria and mtDNA. Perhaps the organization of the mitochondrial genome, and the processes by which it transcribes and replicates, might contribute more to our understanding of the diversity of rates of evolution across major animal groups.

\section{Acknowledgements}

We thank the following for access to samples: modern: Charles Daugherty and Stephen Sarre; ancient: Trevor Worthy, Alan Tennyson (Museum of New Zealand), Neville Hudson (Geology Museum, University of Auckland), Paul Scofield (Canterbury 
Museum), Karl Gillies, Lindsay Hazley (Southland Museum), and Brian Gill (Auckland Museum). We also thank Trevor Worthy for his unpublished radiocarbon dates. We are grateful to Judith Robins for independently verifying aDNA sequences and Leon Huynen for help with cloning. We also thank the editor and four anonymous reviewers for their useful comments on the manuscript.

\section{Online Supplementary Material}

Supplemental data showing sample locations and methodology are available online. 



\section{Figure Legend}

Figure 1. Comparison of evolutionary rates of the mitochondrial HVR regions of tuatara with rates estimated from nine other vertebrate species. The evolutionary rate was estimated using 33 ancient and 41 modern sequences of tuatara. We employed Bayesian statistics based on an Markov chain Monte Carlo (MCMC) approach that accounts for various evolutionary parameters such as base composition, population size, rate and model of evolution using the software BEAST [24]. To model the pattern of nucleotide evolution sophisticated models such as HKY and GTR were used. To account for the variation in the rate of evolution among nucleotide positions, a discrete gamma distribution of six rate categories was used. Wide uniform prior distributions were used to avoid any bias due to restricted prior assumptions. For the estimation of the evolutionary rate in tuatara, priors were set to range from $10^{-10}$ to 1 substitution per site per year and in the case of population size from $10^{7}$ to 1 individual. Both strict and relaxed molecular clock models were used for rate estimation and for each model constant as well as exponential population growth assumptions were examined. The rate estimates were sampled once per 1000 cycles from the total MCMC chain length of 10,000,000 after discarding the initial burn-in 1,000,000 steps. The posterior distributions were examined using the software TRACER [25] and the Effective Sample Sizes (ESS) for mean evolutionary rate, population size and posterior likelihood were found to be $>100$ for all the models used. Our analysis shows that the rate of molecular evolution in tuatara HVR regions is 1.56 (highest posterior density intervals HPD) (0.83 2.34) substitutions per nucleotide per million years. Although this result is based on relaxed molecular clock and constant population size assumptions, the rate estimate and 
the highest HPD (yellow area) were similar when varying a number of a priori assumptions (see Table S5 in supplementary material). The evolutionary rate estimates for nine other vertebrates were obtained from previously published studies that used ancient DNA methods and Bayesian MCMC statistics similar to this study [10, 16, 2628]. The rate estimates obtained for ox, horse, moa and cave lion were adjusted for ancient DNA damage. However this does not bias our result (see main text). The Latin name, the length of the hyper-variable regions (bp), sample size and the age of the samples (thousand years) are as follows: Tuatara (Sphenodon punctatus, 460; 76; 0-9), Adélie penguin (Pygoscelis adeliae; 313; 96; 0-7), aurochs (Bos primigenius, 379; 40; 212), Mappin’s moa (Pachyornis mappini; 241; 14; 1-6), bison (Bison bison/Bison priscus; 601; 182; 0-60), brown bear (Ursus arctos; 130; 30; 10-59), cave bear (Ursus spelaeus; 288; 26; 27-80), cave lion (Panthera leo spelaea; 213; 34; 12-62), ox (Bos taurus; 379; 36; 4-6) and horse (Equus caballus; 348; 12; 1-28). Error bars indicate the HPD intervals.

\section{References}

1. Carroll, R.L. (1988) Vertebrate Paleontology and Evolution. W. H. Freeman \& Co., N.Y.

2. Benton, M.J. (2000) Vertebrate Paleaontology, 2nd edition. Backwell Science, Oxford

3. Cree, A. et al. (1990) Absence of daily cycles in plasma sex steroids in male and female tuatara (Sphenodon punctatus), and the effects of acute capture stress on females. Gen. Comp. Endocrinol. 79, $103-113$

4. Cree, A. et al. (1991) Laparoscopy, radiography, and blood analyses as techniques for identifying the reproductive condition of female tuatara. Herpetologica 47, 238-249 
5. Thompson, M.B. and Daugherty, C.H. (1998) Metabolism of tuatara, Sphenodon punctatus. Comp. Biochem. Physiol. A Mol. Integr. Physiol. 119, 519-522

6. Gillooly, J.F. et al. (2005) The rate of DNA evolution: effects of body size and temperature on the molecular clock. Proc. Natl. Acad. Sci. USA 102, 140-145

7. Martin, A.P. and Palumbi, S.R. (1993) Body size, metabolic-rate, generation time, and the molecular clock. Proc. Natl. Acad. Sci. USA 90, 4087-4091

8. Quinn, T.W. (1992) The genetic legacy of mother goose: phylogeographic patterns of lesser snow goose Chen caerulescens caerulescens maternal lineages. Mol. Ecol. 1, 105-117

9. Stoneking, M. et al. (1992) New approaches to dating suggest a recent age for the human mtDNA ancestor. Philos. Trans. R. Soc. Lond. B Biol. Sci. 337, 167-175

10. Lambert, D.M. et al. (2002) Rates of evolution in ancient DNA from Adelie penguins. Science 295, 2270-2273

11. Ho, S.Y.W. et al. (2005) Time dependency of molecular rate estimates and systematic overestimation of recent divergence times. Mol. Biol. Evol. 22, 1561-1568

12. Briggs, A.W., et al. (2007) Patterns of damage in genomic DNA sequences from a Neandertal. Proc. Natl. Acad. Sci. USA 104, 14616-21

13. Binladen, J. et al. (2006) Assessing the fidelity of ancient DNA sequences amplified from nuclear genes. Genetics 172, 733-41

14. Gilbert, M.T. et al. (2003) Characterization of genetic miscoding lesions caused by postmortem damage. Am. J. Hum. Genet. 72, 48-61

15. Hofreiter, M. et al. (2001) DNA sequences from multiple amplifications reveal artifacts induced by cytosine deamination in ancient DNA. Nucleic Acids Res. 29, 4793-4799

16. Green, R.E. et al. (2006) Analysis of one million base pairs of Neanderthal DNA. Nature 444, 330336

17. Brotherton, P. et al. (2007) Novel high-resolution characterization of ancient DNA reveals C > Utype base modification events as the sole cause of post mortem miscoding lesions. Nucleic Acids Res. 35, 5717-5728 
18. Yang, Z. (1997) PAML: a program package for phylogenetic analysis by maximum likelihood. Comput. Appl. Biosci. 13, 555-556

19. Janke, A. et al. (2001) The mitochondrial genomes of the iguana (Iguana iguana) and the caiman (Caiman crocodylus): implications for amniote phylogeny. Proc. Biol. Sci. 268, 623-631

20. Wu, C.I. and Li, W.H. (1985) Evidence for higher rates of nucleotide substitution in rodents than in man. Proc. Natl. Acad. Sci. USA 82, 1741-1745

21. Holder, M.T. et al. (1999) Two living species of coelacanths? Proc. Natl. Acad. Sci. USA 96, 1261612620

22. Avise, J.C. et al. (1994) A speciational history of living fossils - molecular evolutionary patterns in horseshoe crabs. Evolution 48, 1986-2001

23. Kimura, M. (1983) The Neutral Theory of Molecular Evolution. Cambridge University

24. Drummond, A.J. and Rambaut, A. (2003) BEAST. Oxford: University of Oxford

25. Rambaut, A. and Drummond, A.J. (2004) Tracer. Oxford: University of Oxford.

26. Shapiro, B. et al. (2004) Rise and fall of the Beringian steppe bison. Science 306, 1561-1565

27. Saarma, U. et al. (2007) Mitogenetic structure of brown bears (Ursus arctos L.) in northeastern Europe and a new time frame for the formation of European brown bear lineages. Mol. Ecol. 16, 401-413

28. Edwards, C.J. et al. (2007) Mitochondrial DNA analysis shows a Near Eastern Neolithic origin for domestic cattle and no indication of domestication of European aurochs. Proc. Bio.l Sci. 274, 13771385 